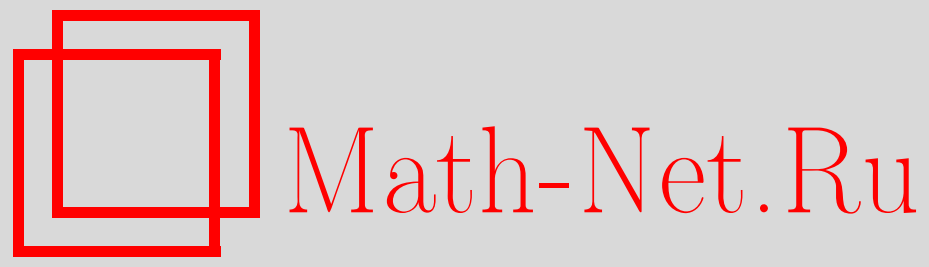

T. M. Suidan, Convex Minorants of Random Walks and Brownian Motion, Теория вероятн. и ее примен., 2001, том 46, выпуск 3, 498-512

DOI: https://doi.org/10.4213/tvp3898

Использование Общероссийского математического портала Math-Net.Ru подразумевает, что вы прочитали и согласны с пользовательским соглашением

http://www . mathnet.ru/rus/agreement

Параметры загрузки:

IP : 3.95 .254 .165

26 апреля 2023 г., 13:08:57

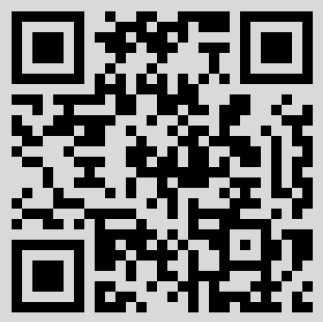




\title{
CONVEX MINORANTS OF RANDOM WALKS AND BROWNIAN MOTION
}

\begin{abstract}
Пусть $\left(S_{i}\right)_{i=0}^{n}$ - процесс случайного блуждания, порожденный последовательностью независимых и одинаково распределенных вещественнозначных случайных величин $\left(X_{i}\right)_{i=1}^{n}$, имеющих плотность. Изучаются вероятностные распределения, связанные с ассоциированным процессом выпуклой миноранты. В частности, исследуется длина самого длинного сегмента выпуклой миноранты. Используя теорию случайных перестановок, мы полностью характеризуем распределение длины $r$-го по величине сегмента выпуклой миноранты броуновского движения на конечных интервалах; мы также указываем явный вид плотности совместного распределения $r$ первых по длине сегментов. Кроме того, мы используем развитые здесь методы для доказательства формулы (E. Sparre Andersen, [9]), позволяющей вычислить вероятность того, что выпуклая миноранта случайного блуждания длины $N$ будет состоять из $m$ сегментов. Приводятся аналогичные утверждения для случайных блужданий со случайными приращениями времени. Эти результаты недавно использованы автором для изучения динамики одномерных частиц с прилипанием.
\end{abstract}

Ключевые слова и фразы: случайное блуждание, выпуклая миноранта, броуновское движение, случайные перестановки.

1. Introduction. In this paper we study properties of convex minorants of random walks generated by real-valued independent identically distributed (i.i.d.) random variables with densities. The convex minorant of any continuous function $f$ on an interval $[0, t]$ is defined to be the greatest convex function $g$ on $[0, t]$ such that $g \leqslant f$. The main results of this paper are:

(i) For a random walk of length $N$, the probability that the associated convex minorant contains a segment of length $n$ is $n^{-1}$ for $n>N / 2$. The expected number of segments of length $n$ is $n^{-1}$.

(ii) For $s \in[0,1]$ and any positive integer $r$, the probability that the length of the $r$-th longest segment, $\mathscr{L}_{W}^{r}$, of the convex minorant generated by Brownian motion on $[0,1]$ is less than $s$ is $F_{r}(s)$, where $F_{r}(s)$ has an explicit formula; the joint density for the lengths of the $r$ longest segments,

${ }^{*}$ PACM-Fine Hall, Princeton University, Princeton, NJ 08544, USA; e-mail: tmsuidan@math.princeton.edu 
$p_{r}\left(x_{1}, \ldots, x_{r}\right)$, can be explicitly given in terms of elementary functions. The expected number of segments of length $l$ with $1 \geqslant t \geqslant l \geqslant s>0$ is $\ln (t / s)$.

(iii) For a random walk of length $N$, the probability that the associated convex minorant is composed of $m$ segments is eqaul to $\left.(m ! N !)^{-1} \frac{d^{N}}{d z^{N}}(-\ln (1-z))^{m}\right|_{z=0}$. The expected number of segments is $\sum_{i=1}^{N} i^{-1}$. The variance of the number of segments is $\sum_{i=1}^{N}\left(1 / i-1 / i^{2}\right)$.

In each of these statements the length of a segment is the projected length on $[0, N]$ or $[0,1]$ depending on the context. Clearly there cannot be more than one segment of length $n>N / 2$ for the random walk. Therefore, the probability in (i) applies to the length of the longest segment of the convex minorant. Charles Goldie pointed out that (iii) was already known to E. Sparre Andersen [9] by different methods.

The structure of the paper is as follows. In Section 2 we prove several properties of sequences of real numbers. The useful probabilistic corollary which follows from these statements is: The probability that a random walk of length $N$ remains above the line which connects the origin to its endpoint is $1 / N$; a conditional version of this statement is also given. M. Steele pointed out that Baxter [2] and Spitzer [10] already knew this statement, however, for completeness we include a full proof. In Section 3 we calculate an auxiliary quantity which is used in Section 4 to prove (i). In Section 5 we use random permutations and the remarkable asymptotic results of Shepp and Lloyd [7] and Vershik and Shmidt [12] to prove (ii). In Section 6 we prove (iii) and discover a connection with the Stirling numbers of the first kind. Section 7 addresses similar issues for random walks with random time increments. It is important to note that the random variables generating the random walks we consider need not have first moments except in Section 5, where we require second moments. In addition, the results of this paper hold in the case that the random variables generating the random walks have continuous distributions, not just densities; however, for simplicity the results are stated for random variables having densities.

The methods used to calculate these quantities have been of use in the author's recent work on one-dimensional adhesion dynamics [11].

2. Several theorems on finite sequences of real numbers. We begin by defining several notions. Let $T: \mathbf{R}^{n} \rightarrow \mathbf{R}^{n}$ be the cyclic shift operator $T\left(x_{1}, \ldots, x_{n}\right)=\left(x_{2}, \ldots, x_{n}, x_{1}\right)$. Note that $T$ is a bijection and denote $T^{m}=T \circ \cdots \circ T m$ times. For $a=\left(a_{1}, \ldots, a_{n}\right) \in \mathbf{R}^{n}$ the walk generated by $a, R_{t}(a)$, is defined as follows: $R_{0}(a)=0$; if $t \in\{1, \ldots, n\}$, $R_{t}(a)=\sum_{i=1}^{t} a_{i} ;$ finally, if $t \in(0, n), R_{t}(a)=R_{\lfloor t\rfloor}(a)+(t-\lfloor t\rfloor) a_{\lfloor t\rfloor+1}$, where $\lfloor t\rfloor$ denotes the greatest integer less than $t$. We define the $m$-th cyclic shift of the walk generated by $a \in \mathbf{R}^{n}, R_{t}(a)$, by $R_{t}\left(T^{m}(a)\right)$. Let $\mu$ be a positive $T$-invariant Borel measure on $\mathbf{R}^{n}$ which is absolutely continuous with respect to Lebesgue measure and define for $r>0$ the positive Borel 
measure $\mu^{r}$ by $\mu^{r}(C)=\mu\left(C \cap B_{r}\right)$, where $B_{r}$ is the open ball of radius $r$ centered about the origin and $C$ is any Borel set; note that $\mu^{r}$ is also $T$-invariant. Define $A=\left\{a=\left(a_{1}, \ldots, a_{n}\right) \in \mathbf{R}^{n}: R_{t}(a)>(t / n) R_{n}(a), \forall t \in\right.$ $(0, n)\}$. For $\alpha \in \mathbf{R}$, let $\mathscr{B}_{\alpha}=\left\{a=\left(a_{1}, \ldots, a_{n}\right) \in \mathbf{R}^{n}: \sum_{i=1}^{n} a_{i}=\alpha\right\}$. We state two lemmas (whose proofs are relegated to the end of this section) followed by several theorems and their probabilistic corollaries.

Lemma 1. If $0 \leqslant j \neq l<n$, then $T^{j}(A) \cap T^{l}(A)=\varnothing$.

Lemma 2. $\mu\left\{\left(\cup_{i=0}^{n-1} T^{i}(A)\right)^{c}\right\}=0$.

Theorem 1. For $\infty>r>0, \mu^{r}$ is a finite measure, $\mu^{r}(A) / \mu^{r}\left(\mathbf{R}^{n}\right)=$ $n^{-1}$, and $\lim _{r \rightarrow \infty}\left(\mu^{r}(A) / \mu^{r}\left(\mathbf{R}^{n}\right)\right)=n^{-1}$. In particular, if $\mu$ is a probability measure then $\mu(A)=n^{-1}$.

P r o of. Simple consequence of Lemma 1, Lemma 2, and the fact that $\mu$ is $T$-invariant.

Corollary 1. If $X_{1}, \ldots, X_{n}$ are real-valued i.i.d. random variables with densities, then $\mathbf{P}\left\{\sum_{j=1}^{i} X_{j}>(i / n) \sum_{j=1}^{n} X_{j}, \forall i \in\{1, \ldots, n-1\}\right\}=n^{-1}$, where $\mathbf{P}$ is the joint probability measure generated by $X_{1}, \ldots, X_{n}$. Note that there is no assumption on the existence of moments.

$\mathrm{P} \mathbf{r}$ o o f. It is enough to check that the product measure on $\mathbf{R}^{n}$ generated by the densities of $X_{1}, \ldots, X_{n}$, satisfies the above conditions on $\mu$.

Let $g \in L_{\text {loc }}^{1}\left(\mathbf{R}^{n}\right)$ be the density of $\mu$ with respect to Lebesgue measure and define the essential support of $\mu$, ess $(\mu)$, in the following way. Let $\Omega$ be the collection of open subsets $\omega \subset \mathbf{R}^{n}$ such that $g(x)=0$ for $\mu$-almost every $x \in \omega$; let $\omega^{*}=\cup_{\omega \in \Omega} \omega$; ess $(\mu)=\left(\omega^{*}\right)^{c}$. We will now define the conditional measure of $A$ with respect to $\mathscr{B}_{\alpha}, \mu\left(A \mid \mathscr{B}_{\alpha}\right)$. Let $\mathscr{A}_{\mu}$ be the set of $\alpha \in \mathbf{R}$ such that $\operatorname{ess}(\mu) \cap \mathscr{B}_{\alpha} \cap(\partial(\operatorname{ess}(\mu)))^{c} \neq \varnothing$. For $\alpha \notin \mathscr{A}_{\mu}$, set $\mu\left(A \mid \mathscr{B}_{\alpha}\right)=0$; for $\alpha \in \mathscr{A}_{\mu}$ define $\mu\left(A \mid \mathscr{B}_{\alpha}\right)=\lim _{\varepsilon \rightarrow 0} \mu_{\varepsilon}\left(A \mid \mathscr{B}_{\alpha}\right)$, where $\mu_{\varepsilon}\left(A \mid \mathscr{B}_{\alpha}\right)=\lim _{r \rightarrow \infty}\left[\mu^{r}\left(A \cap \mathscr{B}_{\alpha}^{\varepsilon}\right) / \mu^{r}\left(\mathscr{B}_{\alpha}^{\varepsilon}\right)\right]$ and $\mathscr{B}_{\alpha}^{\varepsilon}=\left\{a=\left(a_{1}, \ldots, a_{n}\right) \in\right.$ $\left.\mathbf{R}^{n}:\left|\alpha-\sum_{i=1}^{n} a_{i}\right|<\varepsilon\right\}$. As we shall see in the proof of the next theorem these limits exist.

Theorem 2. If $\alpha \in \mathscr{A}_{\mu}$ then $\mu\left(A \mid \mathscr{B}_{\alpha}\right)=n^{-1}$.

P r o of. We make several observations: (i) $T \mathscr{B}_{\alpha}^{\varepsilon}=\mathscr{B}_{\alpha}^{\varepsilon}$; (ii) since $x \in B_{r}$ if and only if $T x \in B_{r}$ and $\mu^{r}$ is $T$-invariant, we have $\mu^{r}\left(T \mathscr{B}_{\alpha}^{\varepsilon}\right)=$ $\mu^{r}\left(\mathscr{B}_{\alpha}^{\varepsilon}\right)$; (iii) if $0 \leqslant j \neq l<n$, Lemma 1 gives that $T^{j}\left(A \cap \mathscr{B}_{\alpha}^{\varepsilon}\right)=T^{j}(A) \cap \mathscr{B}_{\alpha}^{\varepsilon}$ and $T^{l}\left(A \cap \mathscr{B}_{\alpha}^{\varepsilon}\right)=T^{l}(A) \cap \mathscr{B}_{\alpha}^{\varepsilon}$ imply that $T^{j}\left(A \cap \mathscr{B}_{\alpha}^{\varepsilon}\right) \cap T^{l}\left(A \cap \mathscr{B}_{\alpha}^{\varepsilon}\right)=\varnothing$; (iv) since $\mu^{r}$ is $T$-invariant, $\mu^{r}\left(T^{j}\left(A \cap \mathscr{B}_{\alpha}^{\varepsilon}\right)\right)=\mu^{r}\left(T^{l}\left(A \cap \mathscr{B}_{\alpha}^{\varepsilon}\right)\right)$; (v) $\mu^{r}\left(\mathscr{B}_{\alpha}^{\varepsilon} \cap\right.$ $\left.\left(\cup_{i=0}^{n-1} T^{i}\left(A \cap \mathscr{B}_{\alpha}^{\varepsilon}\right)\right)^{c}\right) \leqslant \mu^{r}\left(\left(\cup_{i=0}^{n-1} T^{i}(A)\right)^{c}\right)=0$ by Lemma 2 .

We conclude that for $\alpha \in \mathscr{A}_{\mu}, \varepsilon>0$, and sufficiently large $r, \mu^{r}(A \cap$ $\left.\mathscr{B}_{\alpha}^{\varepsilon}\right)=n^{-1} \mu^{r}\left(\mathscr{B}_{\alpha}^{\varepsilon}\right)$. We see that the above limits exist and the desired result follows.

Corollary 2. Let $X_{1}, \ldots, X_{n}$ be real-valued i.i.d. random variables with densities. Let $\mathbf{P}$ be the joint probability distribution generated by $X_{1}, \ldots, X_{n}$ 
and $\mathbf{P}^{\prime}$ the measure on $\mathbf{R}^{n}$ induced by $\mathbf{P}$. For all $\alpha \in \mathscr{A}_{\mathbf{P}^{\prime}}, \mathbf{P}\left\{\sum_{j=1}^{i} X_{j}>\right.$ $\left.(i / n) \sum_{j=1}^{n} X_{j}, \forall i \in\{1, \ldots, n-1\} \mid \sum_{j=1}^{n} X_{j}=\alpha\right\}=n^{-1}$.

$\mathrm{P} \mathrm{r}$ o of. We need only check that the induced probability measure on $\mathbf{R}^{n}, \mathbf{P}^{\prime}$, satisfies the hypothesis of Theorem 2. This is straightforward.

One should think of Theorem 2 as a statement about the conditional measures of $A$ conditioned on the family of hyperplanes $\mathscr{B}_{\alpha}$.

For $a \in \mathbf{R}^{n}, R_{t}(a)$ is a continuous function on $[0, n]$. The convex minorant of $R_{t}(a)$ is defined as in the introduction and denoted by $\operatorname{co}(a)$. We are now ready to prove Lemma 1 and Lemma 2 .

P r o of of L e m m a 1. Define $E=\left\{a=\left(a_{1}, \ldots, a_{n}\right) \in \mathbf{R}^{n}: \exists i \in\right.$ $\left.\{1, \ldots, n-1\}, \exists m \in\{0,1, \ldots, n-1\}, R_{i}\left(T^{m} a\right)=(i / n) R_{n}(a)\right\}$. Since $E$ is a finite union of codimension 1 submanifolds of $\mathbf{R}^{n}$ the Lebesgue measure of $E$ is zero which implies that $\mu(E)=0$. Letting $F=E^{c}$, we will show that $F=\bigcup_{j=0}^{n-1} T^{j} A$ and that for each $a \in F$ there is exactly one $0 \leqslant j<n$ depending on $a$ such that $T^{j} a \in A$. This, coupled with the fact that $T$ is a bijection, will complete the proof of the lemma. If $a \in A$ the following calculation shows that $T^{m} a \notin A$ for $m=1, \ldots, n-1$ :

$$
\begin{aligned}
\frac{R_{m}(a)}{m} & >\frac{R_{n}(a)}{n} \Longleftrightarrow \frac{R_{m}(a)}{n-m}>\frac{m}{n(n-m)} R_{n}(a) \\
& \Longleftrightarrow \frac{R_{m}(a)}{n-m}>\left(\frac{1}{n-m}-\frac{1}{n}\right) R_{n}(a) \\
& \Longleftrightarrow \frac{1}{n-m}\left(R_{n}(a)-R_{m}(a)\right)<\frac{R_{n}(a)}{n} \\
& \Longleftrightarrow \frac{1}{n-m} R_{n-m}\left(T^{m} a\right)<\frac{R_{n}(a)}{n} .
\end{aligned}
$$

We conclude that for $a \in F$ there exists at most one $j \in\{0, \ldots, n-1\}$ such that $T^{j} a \in A$. We need only give an algorithm for finding the $j \in$ $\{0,1, \ldots, n-1\}$ so that $T^{j} a \in A$. Define $s(a)=R_{n}(a) / n$ and consider the parameterized family of lines in $\mathbf{R}^{2}, y_{c}^{a}(x)=s(a) x+c$. Define $\mathscr{C}_{a}$ to be the set of $c \in \mathbf{R}$ such that $y_{c}^{a}(x)$ lies below $\operatorname{co}(a)$ for $x \in[0, n)$ and let $c^{*}=\sup _{c \in \mathscr{C}_{a}} c$. Since $a \in F$ the line $y_{c^{*}}(x)$ lies below co $(a)$ except at one $t_{a} \in[0, n)$, where it intersects co $(a)$. Clearly $t_{a} \in\{0,1, \ldots, n-1\}$ because $R_{t}(a)$ is a piecewise linear function with «corners» at integer points. At this point a simple geometric argument which can easily be formalized will show that $T^{t_{a}} a \in A$. If $t_{a}=0$ then $a \in A$ as can be seen from drawing a picture (at no time does the path $R_{t}(a)$ intersect the line segment connecting the origin to $\left(n, R_{n}(a)\right) \in \mathbf{R}^{2}$; this is exactly the definition of $\left.a \in A\right)$. If $t_{a} \neq 0$ then partition $R_{t}(a)$ into two pieces at $t_{a}$. We refer to these as the left and right pieces. Attach the right piece to the origin and the left piece to the end of the right piece. This corresponds to constructing the walk generated by $T^{t_{a}} a, R_{t}\left(T^{t_{a}} a\right)$. This walk has the property that it does not cross the 
line connecting the origin to $\left(0, R_{n}(a)\right)$. Thus $T^{t_{a}} a \in A$ and the proof of Lemma 1 is complete.

$\mathrm{Pr}$ o of of $\mathrm{Lemma} 2$. Define $E$ as before and recall that $\mu(E)=0$. We have shown that if $a \in F=E^{c}$ then there exists $j \in\{0,1, \ldots, n\}$ so that $T^{j} a \in A$. So, $\left(\cup_{j=0}^{n-1} T^{j} A\right)^{c}=E$, thus $\mu\left(\left(\cup_{j=0}^{n-1} T^{j} A\right)^{c}\right)=0$.

3. A decomposition of $\mathbf{R}^{n}$. Define $E_{u}=\left\{a=\left(a_{1}, \ldots, a_{n}\right) \in\right.$ $\left.\mathbf{R}^{n}: \exists a_{i_{1}}, \ldots, a_{i_{l}}, \beta_{1}, \ldots, \beta_{l}= \pm 1, \sum_{j=1}^{l} \beta_{j} a_{i_{j}}=l u\right\}$ and $F_{u}=E_{u}^{c}$. For $a \in F_{u}$ we introduce the $u$-decomposition of $a$ which partitions $R_{t}(a)$ into a left and right part, one of which may be trivial. By arguments similar to those in the proof of Lemma 1 , there is a unique pair $\left(i, c^{*}\right) \in\{0,1, \ldots, n\} \times \mathbf{R}$ such that $R_{t}(a)>u t+c^{*}$ for all $t \in[0, n] \backslash\{i\}$ and $R_{i}(a)=u i+c^{*}$. In other words, there is a unique line with slope $u$ so that $\operatorname{co}(a)$ lies above the line except at one point $\left(i, R_{i}(a)\right)$, where the line intersects $\operatorname{co}(a)$. Let $I_{u}: F_{u} \rightarrow\{0,1, \ldots, n\}$ assign to each $a \in F_{u}$ the unique intersection time $i$ just described. The $u$-decomposition of $a \in F_{u}$ is the partition of $R_{t}(a)$ at $I_{u}(a)$ into left- and right-hand sides, one of which may be empty or trivial (this happens if $I_{u}(a)=0$ or $n$ ). The right-hand side of this partition satisfies the condition $a_{I_{u}(a)+1}+\cdots+a_{I_{u}(a)+i}>u i$ for $1 \leqslant i \leqslant n-I_{u}(a)$, while the left-hand side of this partition satisfies the condition $-\left(a_{I_{u}(a)}+\cdots+a_{I_{u}(a)-j}\right)>-(j+1) u$ for $0 \leqslant j<I_{u}(a)$. The uniqueness of the $u$-decomposition leads to the following decomposition of $F_{u}: F_{u}=\cup_{j=0}^{n} \mathscr{D}_{j}^{u}, \mathscr{D}_{j}^{u} \cap \mathscr{D}_{l}^{u}=\varnothing$ if $j \neq l$, where

$$
\begin{aligned}
\mathscr{D}_{j}^{u}= & \left\{a \in F_{u}: I_{u}(a)=j\right\} \\
= & \left\{a \in F_{u}: a_{j+1}+\cdots+a_{j+i}>u i, 1 \leqslant i \leqslant n-j,\right. \\
& \left.a_{j}+\cdots+a_{j-l}<(l+1) u, 1 \leqslant l<j\right\} \\
= & \left\{a \in F_{u}: a_{j+1}+\cdots+a_{j+i}>u i, 1 \leqslant i \leqslant n-j\right\} \\
& \bigcap\left\{a \in F_{u}: a_{j}+\cdots+a_{j-l}<(l+1) u, 0 \leqslant l<j\right\} .
\end{aligned}
$$

This partition of $F_{u}$ will help us calculate a useful quantity. Let $X_{1}, \ldots, X_{n}$ be real-valued i.i.d. random variables which have a distribution with density; let $\mathbf{P}$ denote the joint probability measure induced by $X_{1}, \ldots, X_{n}$; we do not assume existence of moments. Define $\mathbf{P}^{\prime}$ to be the joint probability distribution on $\mathbf{R}^{n}$ generated by these random variables. For $u \in \mathbf{R}$, let $G_{j}(u)=\mathbf{P}\left\{X_{1}+\cdots+X_{i}>u i, 0<i \leqslant j\right\}$ for $j=1, \ldots, n$ and set $G_{0}(u)=1$; define $K_{j}(u)=\mathbf{P}\left\{X_{1}+\cdots+X_{i}<u i, 0<i \leqslant j\right\}$ for $j=1, \ldots, n$ and set $K_{0}(u)=1$.

Theorem 3. $\sum_{j=0}^{n} K_{j}(u) G_{n-j}(u)=1$ for every $u \in \mathbf{R}$.

P r o of. Fix $u \in \mathbf{R}$ and let $\mathscr{L}$ denote Lebesgue measure on $\mathbf{R}^{n}$. Since $\mathbf{P}^{\prime} \ll \mathscr{L}, \mathbf{P}^{\prime}\left(E_{u}\right)=0$, so $\mathbf{P}^{\prime}\left(F_{u}\right)=1 ;$ this is due to the fact that $E_{u}$ is the 
finite union of codimension 1 submanifolds (hyperplanes) of $\mathbf{R}^{n}$ and consequently has Lebesgue measure zero. The fact that $F_{u}=\cup_{j=0}^{n} \mathscr{D}_{j}^{u}$ and $\left\{\mathscr{D}_{j}^{u}\right\}_{j=0}^{n}$ are disjoint implies that $\sum_{j=0}^{n} \mathbf{P}\left(\mathscr{D}_{j}^{u}\right)=1$. The following set manipulations prove the theorem:

$$
\begin{aligned}
\mathbf{P}^{\prime}\left(\mathscr{D}_{j}^{u}\right)= & \mathbf{P}^{\prime}\left\{a \in F_{u}: a_{j+1}+\cdots+a_{j+i}>u i, 1 \leqslant i \leqslant n-j\right\} \\
& \times \mathbf{P}^{\prime}\left\{a \in F_{u}: a_{j}+\cdots+a_{j-l}<u(l+1), 0 \leqslant l<j\right\} \\
= & G_{n-j}(u) K_{j}(u) .
\end{aligned}
$$

The first equality follows from the fact that $\mathbf{P}^{\prime}$ is a product measure; the last equality holds because $\mathbf{P}^{\prime}$ is the induced product measure on $\mathbf{R}^{n}$ generated by i.i.d. random variables and is thus invariant under permutation of coordinates. The proof is complete.

4. The longest segment of the convex minorant generated by random walks of length $N$. Let $X_{1}, \ldots, X_{N}$ be real-valued i.i.d. random variables with densities. For $n>N / 2$ we calculate the probability $L_{n}$ that the longest segment of the convex minorant associated to the random walk generated by $X_{1}, \ldots, X_{N}$ is of length $n$. Let $p_{n}(u)$ be the density for the random variable $\left(X_{1}+\cdots+X_{n}\right) / n$.

Theorem 4. $L_{n}=n^{-1}$ for $n>N / 2$.

P $\mathrm{r}$ o of. The probability that a segment of length $n$ begins at coordinate $j \leqslant N-n$ is

$$
\begin{aligned}
& \int_{\mathbf{R}} d u p_{n}(u)( \mathbf{P}\left\{\sum_{l=1}^{i} X_{j+l}>u i, 1 \leqslant i<n \mid \frac{1}{n} \sum_{l=1}^{n} X_{j+l}=u\right\} \\
& \times \mathbf{P}\left\{\sum_{s=0}^{r} X_{j-s}<u(r+1), 0 \leqslant r<j\right\} \\
&\left.\times \mathbf{P}\left\{\sum_{t=1}^{m} X_{j+n+t}>u m, 1 \leqslant m \leqslant N-(j+n)\right\}\right) \\
&=\int_{\mathbf{R}} d u p_{n}(u) n^{-1} K_{j}(u) G_{N-(n+j)}(u) .
\end{aligned}
$$

Since the event of having a segment of the convex minorant of size exactly $n>N / 2$ begin at coordinate $j$ and the event of having such a segment begin at a different coordinate $l$ are disjoint, the probability of having a segment of size exactly $n>N / 2$ is

$$
\begin{aligned}
& \sum_{j=0}^{N-n} \int_{\mathbf{R}} d u p_{n}(u) n^{-1} K_{j}(u) G_{N-(n+j)}(u) \\
& \quad=\frac{1}{n} \int_{\mathbf{R}} d u p_{n}(u) \sum_{j=0}^{N-n} K_{j}(u) G_{N-(n+j)}(u)=\frac{1}{n} \int_{\mathbf{R}} d u p_{n}(u)=\frac{1}{n} .
\end{aligned}
$$


We observe that for $\frac{1}{2}<s<t \leqslant 1$ the probability that there is a segment of the convex minorant of length $n$, where $s N \leqslant n \leqslant t N$, is $\sum_{s N \leqslant n \leqslant t N} n^{-1}$, which tends to $\ln (t / s)$ as $N \rightarrow \infty$. From the proof of Theorem 4 , we also observe the following fact.

Corollary 3. For any $n \in\{1, \ldots, N\}$, the expected number of segments of length $n$ of the convex minorant is $n^{-1}$.

$\mathrm{Pr}$ o of. If we do not insist on the disjointness of the events in the second step of the proof of Theorem 4, our calculation gives an expression for the expected number of segments of length $n$ of the convex minorant associated to the random walks considered.

5. The convex minorant of Brownian motion. We begin this section by recalling the remarkable results of Shepp and Lloyd [7] and Vershik and Shmidt [12] on the asymptotic distributions of cycle lengths of random permutations. Denote the symmetric group on $n$ symbols by $\mathscr{S}_{n}$ and endow it with the uniform probability distribution $\mathbf{P}$. For $\pi \in \mathscr{S}_{n}$, define $\alpha_{i}(\pi), i=1,2, \ldots, n$, to be the number of cycles of $\pi$ of length $i$; we refer to $\left(\alpha_{1}(\pi), \ldots, \alpha_{n}(\pi)\right)$ as the cycle type of $\pi$. Let $L_{r}^{n}(\pi)$ be the length of the $r$-th longest cycle of $\pi \in \mathscr{S}_{n}$ with the convention that $L_{r}^{n}(\pi)=0$ if $\pi$ has less than $r$ cycles. It is easy to see that $\mathbf{P}\left\{\pi \in \mathscr{S}_{n}: \alpha_{1}(\pi)=a_{1}, \ldots\right.$, $\left.\alpha_{n}(\pi)=a_{n}\right\}=\prod_{j=1}^{n}\left[(1 / j)^{a_{j}} / a_{j} !\right]$. As we will see in the next section, this is exactly the probability that the associated convex minorant of a random walk of length $n$ is composed of $a_{1}$ segments of length $1, a_{2}$ segments of length 2, and so on; this induces a simple bijection between the cycle types of $\mathscr{S}_{n}$ and the segment length composition classes (irrespective of ordering) of the convex minorant of random walks of length $n$. This bijection will soon be exploited. Actually, the connection between the two objects has a history which is given in the Goldie's paper [4]. Shepp and Lloyd [7] proved that the limit distribution of $L_{r}^{n} / n$ can be explicitly described by the following distribution function $F_{r}(\xi)$ on $[0, \infty)$ defined by

$$
\begin{array}{ll}
F_{r}(\xi)=1-\sum_{p=r}^{p<\xi^{-1}} \frac{(-1)^{p-r}}{(r-1) !(p-r) ! p} \int_{A_{p}(\xi)} \cdots \int \frac{d u_{1} \cdots d u_{p}}{u_{1} \cdots u_{p}} & \text { if } 0<\xi<\frac{1}{r}, \\
F_{r}(\xi)=1 & \text { if } \frac{1}{r} \leqslant \xi<\infty,
\end{array}
$$

where $A_{p}(\xi)$ is the region of integration defined by $u_{1}, \ldots, u_{p} \geqslant 1$ and $u_{1}+\cdots+u_{p} \leqslant \xi^{-1}$. We can say even more. Vershik and Shmidt [12] proved that the limit distribution of $\left(L_{1}^{n} / n, \ldots, L_{r}^{n} / n\right)$ exists and has a density $p_{r}\left(x_{1}, \ldots, x_{r}\right)$ on $\Lambda_{r}=\left\{x \in \mathbf{R}^{r}: x_{1} \geqslant \cdots \geqslant x_{r} \geqslant 0, \sum_{i=1}^{r} x_{i} \leqslant 1\right\}$ given by $\left(x_{1} \cdots x_{r-1} \bar{x}_{r}\right)^{-1} f\left(x_{r} / \bar{x}_{r}\right)$, where $f(\xi)=d F_{1}(\xi) / d \xi$ and $\bar{x}_{i}=$ $1-x_{1}-\cdots-x_{i-1}$.

We briefly recall the notions of Brownian motion, Wiener measure, and Donsker approximation. Let $C[0,1]$ be the Banach space of continuous func- 
tions on $[0,1]$ equipped with the standard supremum norm; define $\mathscr{C}$ to be the Borel sigma-algebra of $C[0,1]$ and $W$ to be the standard Wiener measure on the measurable space $(C[0,1], \mathscr{C})[3]$. Define the scaled random walk processes $Y_{t}^{N}$ on $[0,1]$ by $Y_{t}^{N}=\left(S_{\lfloor N t\rfloor}+(N t-\lfloor N t\rfloor) X_{\lfloor N t\rfloor+1}\right) / \sqrt{N \mathbf{E} X_{1}^{2}}$, where $S_{i}=\sum_{j=1}^{i} X_{j}$ and $X_{1}, \ldots, X_{N}$ are as before and have second moment. The theory of weak convergence of measures on metric spaces and Donsker approximation [3] facilitates the computation of several interesting quantities. If $A \in \mathscr{C}$ and $W(\partial A)=0$, then $W_{N}(A) \rightarrow W(A)$ as $N \rightarrow \infty$; here, $W_{N}$ denotes the measures on $(C[0,1], \mathscr{C})$ induced by the scaled random walk processes $Y_{t}^{N}$ and $\partial A$ denotes the boundary of $A$ with respect to the metric space topology of $C[0,1]$.

For $s \in[0,1]$ define $\mathscr{M}_{r}^{s}$ to be the set of functions, $\omega \in C[0,1]$, such that the length of the $r$-th longest segment of the convex minorant of $\omega$ takes a value in $(0, s]$. Note that $W\left(\partial \mathscr{M}_{r}^{s}\right)=0$; a proof of this fact is sketched in the appendix. We calculate $W\left(\mathscr{M}_{r}^{s}\right)$ by simply computing $\lim _{N \rightarrow \infty} W_{N}\left(\mathscr{M}_{r}^{s}\right)$. By the above discussion of random permutations and convex minorants and a simple scaling argument we conclude that

$$
F_{r}(s)=\lim _{N \rightarrow \infty} \mathbf{P}\left\{\frac{L_{r}^{N}}{N} \leqslant s\right\}=\lim _{N \rightarrow \infty} W_{N}\left(\mathscr{M}_{r}^{s}\right)=W\left(\mathscr{M}_{r}^{s}\right),
$$

where $F_{r}$ is defined as above. Define $\mathscr{L}_{W}^{r}$ to be the length of the $r$-th longest segment of the convex minorant generated by Brownian motion on $[0,1]$. We have the desired statement.

Theorem 5. For $s \in[0,1], \mathbf{P}\left\{\mathscr{L}_{W}^{r} \in[0, s]\right\}=F_{r}(s)$, where for $\xi \geqslant r^{-1}$, $F_{r}(\xi)=1$ and for $0<\xi<r^{-1}, F_{r}(\xi)$ is defined by

$$
F_{r}(\xi)=1-\sum_{p=r}^{p<\xi^{-1}} \frac{(-1)^{p-r}}{(r-1) !(p-r) ! p} \int_{A_{p}(\xi)} \cdots \int \frac{d u_{1} \cdots d u_{p}}{u_{1} \cdots u_{p}}
$$

where $A_{p}(\xi)$ is defined as above.

This result clearly scales for Brownian motion on any finite interval $[0, T], T<\infty$; the corresponding distribution is $F_{r}(\xi / T)$. We have thus completely characterized the probability distributions for the length of the $r$-th longest segment of the convex minorant of Brownian motion on finite time intervals. This distribution takes a particularly nice form in the case $r=1$ and $s>\frac{1}{2}: F_{1}(s)=1+\ln s$. Similarly, we can use Donsker approximation and the result of Vershik and Shmidt to prove

Theorem 6. The joint distribution of $\left(\mathscr{L}_{W}^{1}, \ldots, \mathscr{L}_{W}^{r}\right)$ has density, $p_{r}\left(x_{1}, \ldots, x_{r}\right)$, on $\Lambda_{r}$ given by

$$
\frac{1}{x_{1} \cdots x_{r-1} \bar{x}_{r}} f\left(\frac{x_{r}}{\bar{x}_{r}}\right)
$$

where $f(\xi)=d F_{1}(\xi) / d \xi$ and $\bar{x}_{i}=1-x_{1}-\cdots-x_{i-1}$. 
This completely characterizes the joint distribution of the lengths of the $r$ longest segments of the convex minorant on $[0,1]$. Just as before, this result scales for Brownian motion on any finite interval $[0, T]$. It is also interesting to note that using Donsker approximation and the results of Section 4 we can compute the expected number of segments in the convex minorant of Brownian motion which have lengths in $[s, t]$, where $0<s<$ $t \leqslant 1$; this expected number is $\ln (t / s)$. To see this we need only mimic the proof of Corollary 3 of Section 4.

Previous research has been done on the problem of the convex minorant generated by Brownian motion (e.g. [1], [5], [6]). This work is devoted to studying other interesting features of the problem such as the distribution of successive slopes and its relation to pure jump processes. Also of interest in this direction is [3].

6. The number of segments composing the convex minorant generated by random walks of length $N$. As has become customary, let $X_{1}, \ldots, X_{N}$ be real-valued i.i.d. random variables with density. We will now use the methods developed in the previous sections to prove a result already known to E. Sparre Andersen [9].

Theorem 7. The probability that the convex minorant associated to the random walk generated by $X_{1}, \ldots, X_{N}$ is composed of exactly $m$ segments is:

$$
\frac{1}{m !} \sum_{\substack{n_{1}+\cdots+n_{m}=N \\ n_{1}, \ldots, n_{m}>0}} \frac{1}{n_{1} \cdots n_{m}}=\left.\frac{1}{m ! N !} \frac{d^{N}}{d z^{N}}\left\{\left(\ln \frac{1}{1-z}\right)^{m}\right\}\right|_{z=0} .
$$

P r o of. We begin with a few definitions. Let $\mathbf{Z}_{m}^{N}=\left\{\left(b_{1}, \ldots, b_{m}\right) \in\right.$ $\left.\mathbf{Z}^{m}: b_{i}>0, b_{1}+\cdots+b_{m}=N\right\}$. Define the equivalence relation $\delta$ on $\mathbf{Z}_{m}^{N}$ to be: $\left(c_{1}, \ldots, c_{m}\right) \delta\left(b_{1}, \ldots, b_{m}\right)$ if and only if $\left(c_{1}, \ldots, c_{m}\right)=\tau\left(b_{1}, \ldots, b_{m}\right)$ for some element $\tau$ of the symmetric group of order $m$. Denote by $\mathscr{H}$ the quotient space $\mathbf{Z}_{m}^{N} / \delta$. Define the multiplicity function $g$ on $\mathscr{H}$ : For $b \in \mathscr{H}, g(b)=$ $\left(r_{1} ! r_{2} ! \cdots r_{j} !\right)^{-1}$, where $r_{1}, \ldots, r_{j}$ are the multiplicities of the coordinates of $b$; for example, if $N=20, m=7$, and a representative of $b \in \mathscr{H}$ is $(4,2,2,5,3,1,3)$, then $g(b)=\frac{1}{2 ! 2 !}=\frac{1}{4}$. Define $P(b)=g(b)\left(b_{1} \cdots b_{m}\right)^{-1}$. Rewriting the first formula of the theorem we find

$$
\begin{aligned}
\frac{1}{m !} \sum_{\substack{n_{1}+\cdots+n_{m}=N \\
n_{1}, \cdots, n_{m}>0}} \frac{1}{n_{1} \cdots n_{m}} & =\frac{1}{m !} \sum_{b \in \mathscr{H}} \frac{1}{b_{1} \cdots b_{m}}(m ! g(b)) \\
& =\sum_{b \in \mathscr{H}} \frac{1}{b_{1} \cdots b_{m}} g(b)=\sum_{b \in \mathscr{H}} P(b) .
\end{aligned}
$$

We now explain the role of $P(b)$ for $b \in \mathscr{H}$. Fix $c \in \mathbf{Z}_{m}^{N}$ and define the $i$-th subwalk of $c$ to be the random walk of length $c_{i}$ which begins at coordinate $c_{1}+\cdots+c_{i-1}$ and ends at coordinate $c_{1}+\cdots+c_{i}$; 
this is just the part of the random walk of length $N$ which is generated by $X_{1+c_{1}+\cdots+c_{i-1}}, \ldots, X_{c_{1}+\cdots+c_{i}}$. Figure 1 at the end of this section is a particular realization of this type of random walk and its subwalks. Let the random variables $s_{1}^{c}, \ldots, s_{m}^{c}$ be the slopes of the lines which connect the end points of the subwalks of $c$. The probability that each of the $m$ subwalks of $c$ lies above the line connecting its endpoints and $s_{1}^{c}<\cdots<s_{m}^{c}$ is $\left(c_{1} \cdots c_{m}\right)^{-1} \mathbf{P}\left\{s_{1}^{c}<\cdots<s_{m}^{c}\right\}$; the product structure is due to the independence of all the events considered. These are exactly the conditions for the convex minorant of the random walk of length $N$ to be composed of $m$ segments of ordered lengths $c_{1}, \ldots, c_{m}$; see Figure 1. Summing over distinct (from the perspective of ordering) permutations of the coordinates of $c$ we get $g(c) /\left(c_{1} \cdots c_{m}\right)$, where $c$ is now considered as an element of $\mathscr{H}$ rather than $\mathbf{Z}_{m}^{N}$. This expression, $P(c)$, is the probability that the convex minorant of the random walk of length $N$ has exactly $m$ segments with lengths $c_{1}, \ldots, c_{m}$ irrespective of order. Summing over all $c \in \mathscr{H}$ we get the last expression of the above calculation and thus the first expression in (1).

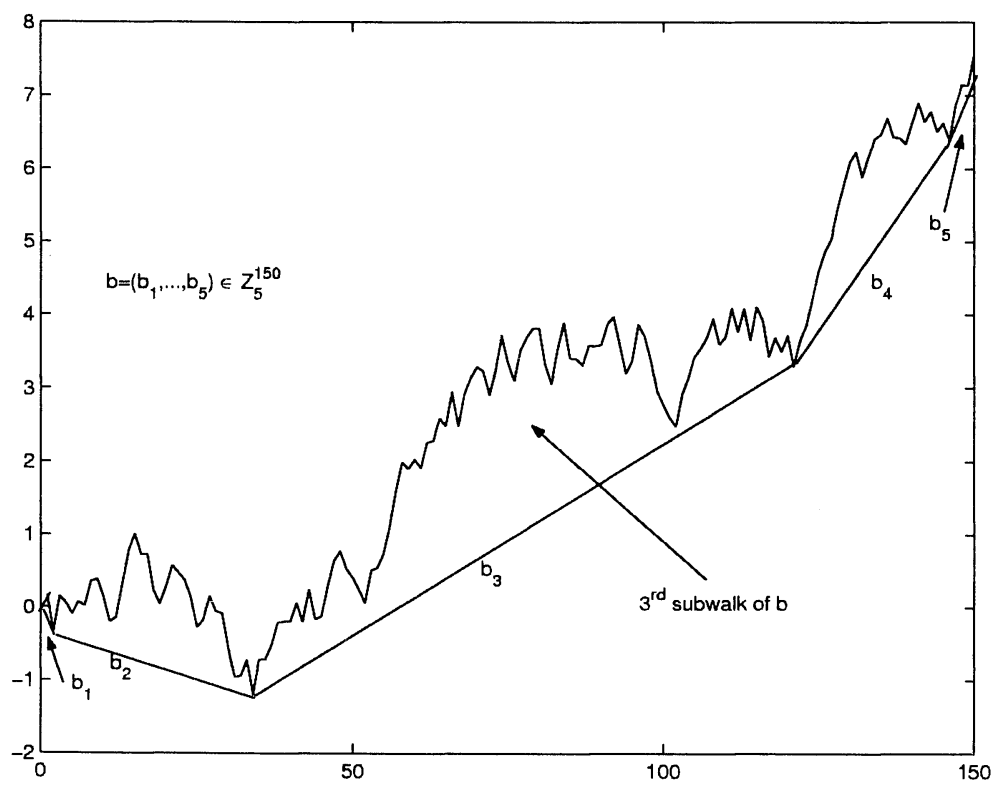

Figure 1. Realization of a random walk and its convex minorant.

Evaluating this expression by using generating functions is straightforward. Let $\xi(z)=\sum_{i=1}^{\infty} z^{i} / i$; note that $\xi(z)=-\ln (1-z)$ for $|z|<1$. Also note that:

$$
(\xi(z))^{m}=\sum_{j=m}^{\infty} z^{j}\left\{\sum_{\substack{i_{1}+\cdots+i_{m}=j \\ i_{1}, \ldots, i_{m}>0}} \frac{1}{i_{1} i_{2} \cdots i_{m}}\right\}=(-\ln (1-z))^{m} .
$$


Recognizing that

$$
\left.\frac{1}{N !} \frac{d^{N}}{d z^{N}}\left\{\left(\ln \frac{1}{1-z}\right)^{m}\right\}\right|_{z=0}=\sum_{\substack{n_{1}+\cdots+n_{m}=N \\ n_{1}, \ldots, n_{m}>0}} \frac{1}{n_{1} n_{2} \cdots n_{m}}
$$

we arrive at the second expression in (1). This completes the proof.

It is interesting to note that

$$
s(N, m)=\left.\frac{N !}{m !} \frac{d^{N}}{d z^{N}}\left\{\left(\ln \frac{1}{1-z}\right)^{m}\right\}\right|_{z=0}
$$

are the Stirling numbers of the first kind which have been studied in number theory and combinatorics. C. Goldie has noted this fact in his review paper [4].

From this theorem we can recover a result of Section 4 .

Corollary 4. The expected number of segments composing the convex minorant associated to the random walk of length $N$ is $\sum_{m=1}^{N} m^{-1}$. The variance of the number of segments is $\sum_{m=1}^{N}\left(m^{-1}-m^{-2}\right)$.

P r o of. We simply compute:

$$
\begin{aligned}
& \left.\sum_{m=1}^{N} m \frac{1}{m ! N !} \frac{d^{N}}{d z^{N}}\left\{\left(\ln \frac{1}{1-z}\right)^{m}\right\}\right|_{z=0} \\
& \quad=\left.\frac{1}{N !} \frac{d^{N}}{d z^{N}}\left\{\left(\ln \frac{1}{1-z}\right) \sum_{m=1}^{N} \frac{1}{(m-1) !}\left(\ln \frac{1}{1-z}\right)^{m-1}\right\}\right|_{z=0} \\
& \quad=\left.\frac{1}{N !} \frac{d^{N}}{d z^{N}}\left\{\left(\ln \frac{1}{1-z}\right)\left(\frac{1}{1-z}-\sum_{m=N}^{\infty} \frac{1}{m !}\left(\ln \frac{1}{1-z}\right)^{m}\right)\right\}\right|_{z=0} \\
& =\left.\frac{1}{N !} \frac{d^{N}}{d z^{N}}\left\{\frac{1}{1-z} \ln \frac{1}{1-z}\right\}\right|_{z=0} \\
& =\frac{1}{N !} \sum_{m=1}^{N}(m-1) !(N-m) !\left(\begin{array}{l}
N \\
m
\end{array}\right)=\sum_{m=1}^{N} \frac{1}{m} .
\end{aligned}
$$

A similar but longer computation gives the formula for the variance.

We conclude that the expected number of segments composing the convex minorant of a random walk of length $N$ grows as $\ln N$.

7. Other related theorems and facts. This section addresses the same questions as in Sections 2, 3, 4, and 6 for random walk processes that have random time increments. We also address questions pertaining to random samplings of Brownian motion and continuous time stochastic processes with stationary independent increments. 
Let $T_{1}, \ldots, T_{N}$ be positive i.i.d. random variables and $X_{1}, \ldots, X_{N}$ i.i.d. random variables with densities. In addition, we assume that all of random variables involved are independent. Define $S_{0}=0, S_{i}=\sum_{j=1}^{i} X_{j}$ for $i=1,2, \ldots, N$, and the random variable $\phi_{t}=\max \left\{m \in \mathbf{Z}: \sum_{j=1}^{m} T_{j}<t\right\}$ for positive $t$. It will be convenient to denote by $T^{N}$ the random variable $\sum_{j=1}^{N} T_{j}$. Define

$$
\begin{array}{ll}
\mathscr{G}_{t}=S_{\phi_{t}}+\frac{t-\sum_{j=1}^{\phi_{t}} T_{j}}{T_{\phi_{t}+1}} X_{\phi_{t}+1} & \text { for } t \in\left[0, T^{N}\right], \\
\mathscr{F}_{t}=\mathscr{G}_{t T^{N}} & \text { for } t \in[0,1] .
\end{array}
$$

$\mathscr{F}_{t}$ is a stochastic process on $[0,1]$. We note that each theorem in this section which is stated for $\mathscr{F}_{t}$ has a natural analogue for $\mathscr{G}_{t}$. In the following we will denote by $\mathbf{P}$ the product probability measure on $\mathbf{R}^{2 N}$ which is generated by the distribution of the random variables $X_{1}, \ldots, X_{N}, T_{1}, \ldots, T_{N}$.

Theorem 8. $\mathbf{P}\left\{\mathscr{F}_{t}>t \mathscr{F}_{1}, \forall t \in(0,1)\right\}=1 / N$.

Theorem 9. If $\alpha \in \mathscr{A}_{P}$, then

$$
\mathbf{P}\left\{\mathscr{F}_{t}>t \mathscr{F}_{1}, \quad \forall t \in(0,1) \mid \mathscr{F}_{1}=\alpha\right\}=\frac{1}{N} .
$$

Here $\mathscr{A}_{\mathbf{P}}$ is defined as in Section 2 with the exception that $\mathscr{B}_{\alpha}$ is replaced by $\mathscr{B}_{\alpha} \times \mathbf{R}^{N}$. In the next two theorems we will condition on the random times as well as the sum of the $X_{i}$ without explicitly worrying about conditions like $\left\langle\alpha \in \mathscr{A}_{\mathbf{P}} \gg\right.$. Technically, these conditions need to be imposed, but for the purpose of this section we will take comfort in the fact that this is straightforward. Define $\sigma\left(t_{1}, \ldots, t_{N}\right)$ to be the set of cyclic permutations of $\left(t_{1}, \ldots, t_{N}\right) \in \mathbf{R}^{N}$.

Theorem 10.

$$
\mathbf{P}\left\{\mathscr{F}_{t}>t \mathscr{F}_{1}, \forall t \in(0,1) \mid\left(T_{1}, \ldots, T_{N}\right) \in \sigma\left(t_{1}, \ldots, t_{N}\right)\right\}=\frac{1}{N} .
$$

Theorem 11. P $\left\{\mathscr{F}_{t}>t \mathscr{F}_{1}, \forall t \in(0,1) \mid\left(T_{1}, \ldots, T_{N}\right) \in \sigma\left(t_{1}, \ldots, t_{N}\right)\right.$, $\left.\mathscr{F}_{1}=\alpha\right\}=1 / N$.

The proofs of these theorems resemble those in Section 2. They rely on the fact that it is possible to consider the convex minorants associated to random walks and cyclic shifts of these walks.

Fix positive $t_{1}, \ldots, t_{N}$ and define $G_{j}(u)=\mathbf{P}^{X_{1}, \ldots, X_{N}}\left\{X_{1}+\cdots+X_{i}>\right.$ $\left.u\left(t_{1}+\cdots+t_{i}\right), \forall i \in\{1,2, \ldots, j\}\right\}$ for $j=1,2, \ldots, N$ and $G_{0}(u)=1$; define $K_{j}(u)=\mathbf{P}^{X_{1}, \ldots, X_{N}}\left\{X_{1}+\cdots+X_{i}<u\left(t_{1}+\cdots+t_{i}\right), \forall i \in\{1,2, \ldots, j\}\right\}$ for $j=1,2, \ldots, N$ and $K_{0}(u)=1$. Here, $u \in \mathbf{R}$ and $\mathbf{P}^{X_{1}, \ldots, X_{N}}$ is the product probability measure on $\mathbf{R}^{N}$ generated by $X_{1}, \ldots, X_{N}$. A natural modification of the $u$-decomposition of Section 3 gives 
Theorem 12. $\sum_{j=0}^{N} K_{j}(u) G_{N-j}(u)=1$.

If we change our notion of length of a segment we can prove results similar to those in Section 4. Let the degree of a segment of the convex minorant associated to the random walk process $\mathscr{F}_{t}$ be the number of time increments that compose it; this is a natural definition since the time increments of this process which are defined by $T_{1}, \ldots, T_{N}$ are random. Define $H_{n}$ to be the probability that the highest degree of any segment of the convex minorant is $n$. By following the proof in Section 4 we find

Theorem 13. $H_{n}=n^{-1}$ for $n>N / 2$.

This shows that for $\frac{1}{2}<s<t \leqslant 1$ the probability that a segment of degree $r$ belongs to the convex minorant, $s N \leqslant r \leqslant t N$, tends to $\ln (t / s)$ as $N$ tends to infinity.

We mention a fact in the spirit of Section 6 . The probability that exactly $m$ segments compose the convex minorant associated to a random walk having $N$ random increments is

$$
\left.\frac{1}{m ! N !} \frac{d^{N}}{d z^{N}}\left\{\ln \frac{1}{1-z}\right\}^{m}\right|_{z=0} .
$$

The reasoning is similar to that of Section 6. Once again, we emphasize the importance of the conditioning theorem. It shows the independence of the $n^{-1}$ property and the slope of the segment, a crucial fact used in the calculation.

The last result of this nature concerns continuous time stochastic processes having independent, stationary increments. Assume that $\zeta_{t}$ satisfies these conditions and for each $t \zeta_{t}$ has a density. Let $T_{1}, \ldots, T_{N}$ be positive i.i.d. random variables. Let $l_{1}, \ldots, l_{N}$ be the random variables $l_{j}=\sum_{i=1}^{j} T_{i} / \sum_{i=1}^{N} T_{i}$. If $\zeta_{t}$ is such a stochastic process on $[0,1]$, we have

\section{Theorem 14.}

$$
\mathbf{P}\left\{\zeta_{l_{i}}>l_{i} \zeta_{1}, 1 \leqslant i<N \mid\left(l_{1}, \ldots, l_{N}\right) \in \sigma\left(t_{1}, \ldots, t_{N}\right)\right\}=\frac{1}{N} .
$$

Here $\sigma\left(t_{1}, \ldots, t_{N}\right)$ is the set of cyclic shifts of $\left(t_{1}, \ldots, t_{N}\right)$. Once again we emphasize that we need technical conditions imposed on $t_{1}, \ldots, t_{N}$ so that the conditioning makes sense, but these are clear. This statement is proven in a similar way to the theorems at the beginning of this section. It is, however, a different statement and it applies to random (or equidistant) samplings of Brownian motion with or without drift.

8. Concluding remarks. In this paper, we prove a number of facts about convex minorants of random walks generated by i.i.d. random variables with densities. Most of these facts depend on the use of the cyclic shift property described in Section 2. The only other tool is the decomposition of $\mathbf{R}^{n}$ into parts which correspond nicely to random walk paths. We 
completely characterize the length of the $r$-th longest segment of the convex minorant process generated by Brownian motion on finite time intervals as well as the joint densities of the first $r$ longest segments; the asymptotic theorems of the theory of random permutations play a strong role.

The probabilistic facts derived have been useful in the author's work on one-dimensional adhesion dynamics [11] and may be useful in other contexts as well. Some of the many interesting papers on the subject of convex minorants of Brownian motion and random walks are included in the references.

9. Appendix. This appendix discusses the fact that $W\left(\partial \mathscr{M}_{s}^{r}\right)=0$. First, equally partition the unit interval into $2^{n}$ pieces. We will ask the following question: Given that the Brownian paths take values in $\left[-2^{-n}, 2^{-n}\right]$ at time 1 and lie above the line connecting the origin to the end point (at time 1) at each point of the form $i 2^{-n}$ for $i=1, \ldots, 2^{n}-1$, what is the probability that the Brownian paths take values in $\left[-2^{-n}, 2^{-n}\right]$ for some time $j 2^{-n}$, where $j=1,2, \ldots, 2^{n}-1$. This is a simple calculation given our knowledge of finite dimensional distributions of Brownian motion and the results in Section 2:

$$
\begin{array}{r}
\sum_{j=1}^{2^{n}-1} \frac{\frac{1}{j\left(2^{n}-j\right)} \int_{-2^{-n}}^{2^{-n}} \frac{\exp \left(-x^{2} /\left(2 j 2^{-n}\right)\right)}{\sqrt{2 \pi j 2^{-n}}} d x \int_{-2^{-n}}^{2^{-n}} \frac{\exp \left(-y^{2} /\left(2\left(1-j 2^{-n}\right)\right)\right)}{\sqrt{2 \pi\left(1-j 2^{-n}\right)}} d y}{\frac{1}{2^{n}} \int_{-2^{-n}}^{2^{-n}} \frac{\exp \left(-z^{2} / 2\right)}{\sqrt{2 \pi}} d z} \\
\leqslant \sum_{j=1}^{2^{n}-1} \frac{2}{2^{2 n}}\left(\frac{j}{2^{n}}\left(1-\frac{j}{2^{n}}\right)\right)^{-3 / 2} \longrightarrow 0 \text { as } n \rightarrow \infty .
\end{array}
$$

The last expression can be attained from a Riemann sum approximation. This calculation shows us that the above statements are in fact true. When $\partial \mathscr{M}_{s}^{r}$ is examined we see that these are exactly the types of probabilities we wish to consider.

Acknowledgments. The author would like to thank Ya. G. Sinai for helpful comments and advice. The author also wishes to thank M. Aizenman, P. Carmona, R. Carmona, S. Güntürk, H. Kesten, E. Lieb, J. Schenker, M. Yor, and Ö. Yılmaz for helpful discussions and communications.

The author would like to thank C. Goldie for informing him about Sparre Andersen's result which is reproven in Section 6. The author also wishes to thank M. Steele for his interest in this work and his reference to [2], [7], and [10]. My conversations with M. Steele have been very interesting and productive. A discussion on random permutations with A. Borodin was also very productive. 


\section{REFERENCES}

1. Bass R. Markov processes and convex minorants. - Lecture Notes in Math., 1984, v. 1059 , p. $29-41$.

2. Baxter G. A combinatorial lemma for complex numbers. - Ann. Math. Statist., 1961, v. 32, p. 901-904.

3. Биллингсли П. Сходимость вероятностных мер. М.: Наука, 1977, 352 с.

4. Goldie C. Records, permutations, and greatest convex minorants. - Math. Proc. Cambridge Philos. Soc., 1989, v. 106, № 1, p. 169-177.

5. Groeneboom P. The concave majorant of Brownian motion. - Ann. Probab., 1983, v. 11 , № 4 , p. $1016-1027$.

6. Pitman J. Remarks on the convex minorant of Brownian motion. - Progr. Probab. Statist., 1983, v. 5, p. 219-227.

7. Shepp L. A., Lloyd S. P. Ordered cycle lengths in a random permutation. - Trans. Amer. Math. Soc., 1966, v. 121, p. 340-357.

8. Sinai Ya. G. Convex hulls of random processes. - Amer. Math. Soc. Transl., 1998, v. 186 , № 2 , p. 153-159.

9. Sparre Andersen E. On the fluctuations of sums of random variables. II. - Math. Scand., 1954, v. 2, p. 195-223.

10. Spitzer F. A combinatorial lemma and its application to probability theory. - Trans. Amer. Math. Soc., 1956, v. 82, p. 323-339.

11. Suidan T. Adhesion dynamics on the line: The mass aggregation process. - J. Statist. Phys., 2000, v. 101, № 3-4, p. 893-903.

12. Вершик A.M., Шмидт A. A. Предельные меры, возникающие в асимптотической теории симметрических групп. I. - Теория вероятн. и ее примен., 1977, т. 22, B. 1, c. $72-88$.

13. Williams $D$. Path decomposition and continuity of local time for one-dimensional diffusions. I. - Proc. London Math. Soc., Ser. (3), 1974, v. 28, p. 738-768.

Поступила в редакцию 